\title{
PRECISE POSITIONING CONTROL FOR A HYDRAULIC MOTOR SYSTEM WITH DEAD ZONE USING A STATE FEEDBACK NEURAL NETWORK
}

\author{
Hiroshi KATOH ${ }^{*}$, Takao NISHIUMI ${ }^{*}$, and Shizurou KONAMI ${ }^{*}$ \\ * Department of Mechanical Systems Engineering \\ National Defense Academy \\ 1-10-20 Hashirimizu, Yokosuka, Kanagawa, 239-8686 Japan \\ (E-mail: nishiumi@nda.ac.jp)
}

\begin{abstract}
It is generally not easy to acquire high positioning accuracy in hydraulic servo control systems, since they have an intense non-linearity, such as dead zone, which is originally caused by the leakage and friction. It is considered that the Neural Network compensator is one of the available options to get over the inherent problem. This paper deals with the effect of dither/PWM signals on the steady position error in the hydraulic motor drive system with a state feedback Neural Network. From the computer simulation, it is clarified that the application of the dither/PWM signals to the Neural Network control system improves the positioning performance by accompanying the assistance of the Neural Network capability. In addition, the effectiveness of the dither/PWM signals is also verified by experimental results.
\end{abstract}

\section{KEY WORDS}

Hydraulic motor, Position control, Neural Network, Dead zone, Dither, PWM

\section{NOMENCLATURE}

$A$ : Amplitude of signal wave

$B_{v}:$ Viscous coefficient

$c$ : Carrier wave for PWM signal

$C_{v}$ : Volume capacitance

$\Delta d$ : Dither signal

$D_{m}$ : Motor displacement

$f$ : Frequency of signal wave

$J_{m}$ : Moment of inertia

$K_{p}, K_{u}$ : Servovalve flow and pressure gain

$p_{1}, p_{2}$ : Pressures between servovalve and motor

$p_{L}:$ Load pressure $\left(p_{L}=p_{1}-p_{2}\right)$

$p_{s}:$ Supply pressure

$R_{m}$ : Leakage resistance

$T_{D}$ : Driving torque

$T_{f}$ : Load friction torque

$T_{k}$ : Kinetic friction torque

$T_{s}:$ Static friction torque $u$ : Control signal

$v_{r}:$ Reference position input

$v_{o}$ : Output signal from Neural Network

$\Lambda(n)$ : Cost function

$\Pi$ : Evaluation value

$\theta$ : Motor angular position

$\theta_{r}:$ Reference model output

$\omega$ : Motor angular velocity

$\omega_{n}$ : Natural frequency of hydraulic servo motor system

$\zeta$ : Damping ratio of hydraulic servo motor system

Subscripts $d$ : Dither, $\quad p$ : PWM, $s s$ : Steady state

\section{INTRODUCTION}

Hydraulic motors controlled by servovalves are widely utilized as the position and velocity servo control system in all kinds of industrial and vehicle fields, because of their high power density and quick response. However, the hydraulic motor mechanism has inherent unwanted 
problems such as the dead zone and hysterics caused by Coulomb's friction and leakage [1]. The dither signal is well known as one of the classical techniques to improve the static characteristics of the servovalves under the transit condition from static to kinetic friction $[2,3]$. Substituting for the dither system, pulse width modulation effects on the hydraulic servovalves has been investigated $[4,5]$.

In recent years, numerous studies have been conducted to improve this non-linearity of the hydraulic servo system by introducing various control algorithms. The Neural Network can be considered as a fascinating controller because it is capable of flexible learning and mapping. The authors have carried out research on the quasi model reference adaptive position control with a Neural Network compensator for the hydraulic servomotor with friction and leakage $[6,7]$.

The object of this research is to attain precise position accuracy employing the Neural Network state feedback compensator in the hydraulic servo motor system with undesirable dead zone. In this paper, two methods for improving the steady state performance are proposed for the Neural Network control system. One of them is the conventional method using dither signals, which superimpose a high harmonic frequency wave with small amplitude on the system input. One feature this technique offers is that the circuit is easily produced. Here, the sinusoidal dither will only be discussed. The other one is a unique technique using pulse width modulation (PWM) on the actuating signal around the dead zone.

Consequently, the simulation and experimental results will reveal that both techniques respectively assist the online training of the Neural Network while the actuating input with small alternative signals are operated in the steady state.

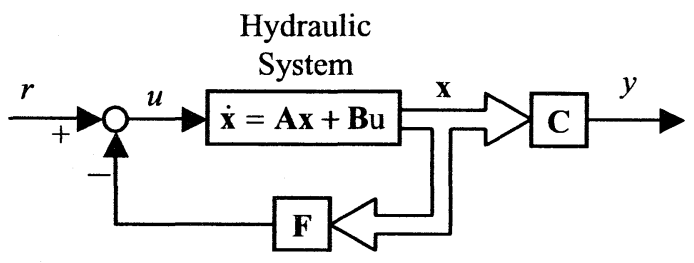

Fig.1 State feedback control system

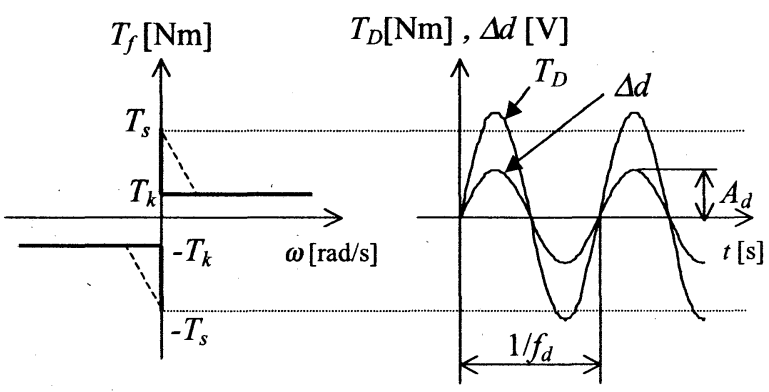

Fig.2 Friction model and dither signal

\section{SYSYEM DESCRIPTION}

In general, the hydraulic servo motor system can be described by three basic equations, that is, the continuity equation with fluid compressibility and internal and external motor leakages, the flow rate equation through the critical lapped spool of the servo valve, and the torque equation considering friction and viscous damping. Therefore, the state-space notation is introduced by linearizing these basic equations [7].

$$
\begin{aligned}
\frac{d \mathbf{x}}{d t} & =\mathbf{A} \mathbf{x}+\mathbf{B} u+\mathbf{d} \\
& =\left[\begin{array}{ccc}
0 & 1 & 0 \\
0 & -B_{v} / J_{m} & D_{m} / J_{m} \\
0 & -D_{m} / C_{v} & -\left(K_{p}+1 / R_{m}\right) / C_{v}
\end{array}\right] \mathbf{x} \\
& +\left[\begin{array}{c}
0 \\
0 \\
K_{u} / C_{v}
\end{array}\right] u+\left[\begin{array}{c}
0 \\
-1 / J_{m} \\
0
\end{array}\right] T_{f} \\
\mathbf{x} & =\left[\begin{array}{lll}
\theta & \omega & p_{L}
\end{array}\right]^{T} \\
y & =\mathbf{C x}=\left[\begin{array}{lll}
1 & 0 & 0
\end{array}\right] \mathbf{x}
\end{aligned}
$$

The block diagram of the closed-loop position control system with the state feedback compensator $\mathbf{F}$ is shown in Fig. 1, when the third term of the right side of the Eq.(1) is omitted.

In the previous report [7], The gain $\mathbf{F}$ of the state feedback compensator was determined by the pole placement method. As a result, the nominal model became the second order system with undamped natural frequency $\omega_{\mathrm{n}}=30 \mathrm{rad} / \mathrm{s}$ and damping ratio $\zeta=1.0$, which was also utilized for the reference model. By means of offline training using simulation data, the feedback characteristics were mapped to the Neural Network, which consists of an input layer with 4 neurons, the first hidden layer with 8 neurons, the second hidden layer with 4 inputs and one output neuron.

\section{POSITION CONTROL SYSTEM CONSIDERING DEAD ZONE}

\subsection{Friction model}

The load friction torque $T_{f}$ is added as the non-linear term on the right side in Eq.(1) as a system disturbance d. This torque commonly depends on the angular velocity known as Coulomb's friction. The left side of Fig. 2 illustrates the approximate model of the static friction torque $T_{s}$ and kinetic friction torque $T_{k}$ that are shown in the following equations.

$$
\begin{aligned}
& \omega=0: T_{f}=\operatorname{sign}\left(p_{L}\right) T_{s} \\
& \omega \neq 0: T_{f}=\operatorname{sign}(\omega) T_{k}
\end{aligned}
$$

The friction characteristics are represented by dashed lines, but it has been experimentally confirmed that the proposed model shown by solid lines is suitable for the 


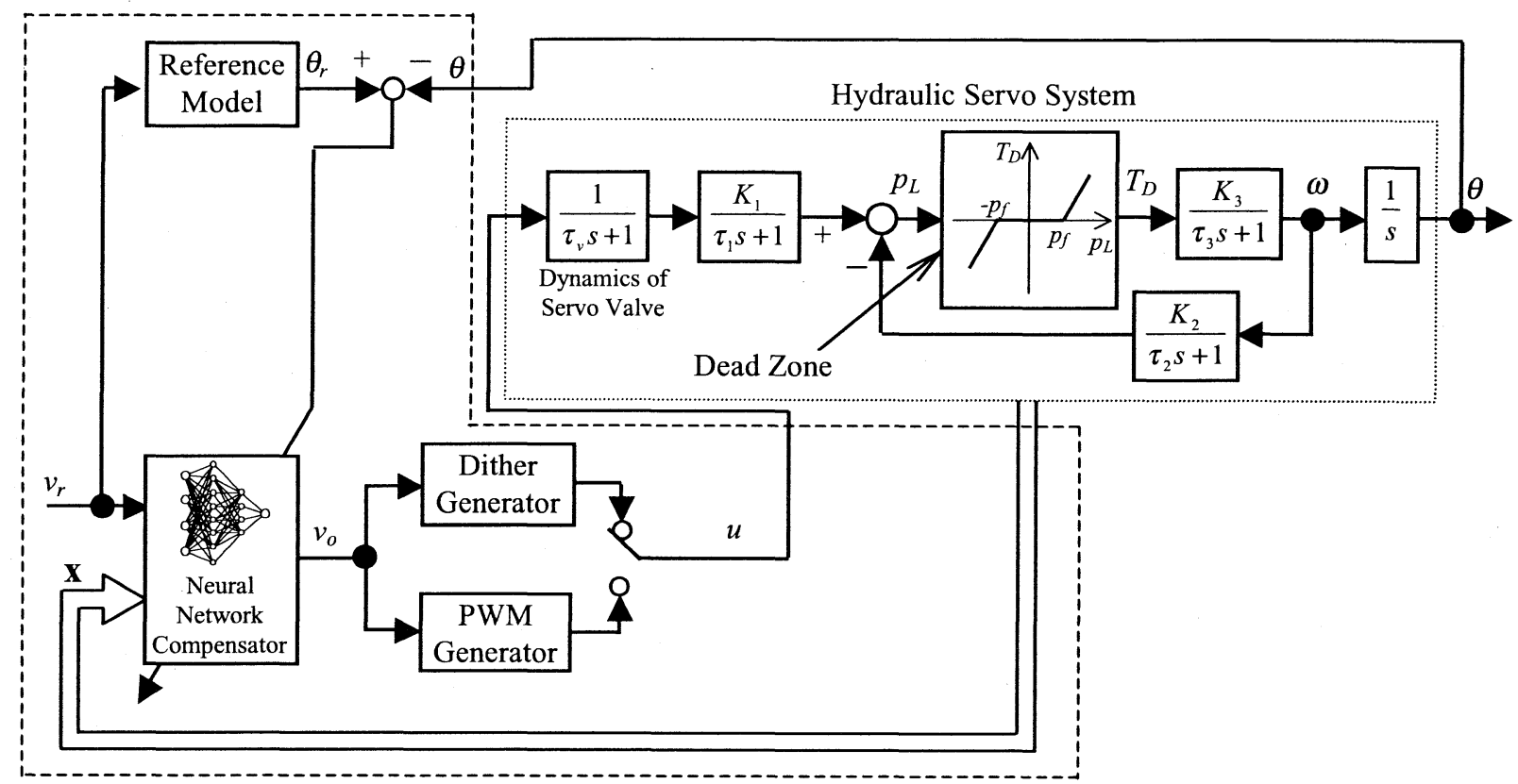

Fig.3 State feedback Neural Network control system using dither/PWM generator

present hydraulic system.

In order to attain highly accurate positioning, the adequate pressure differences $p_{L}$ have to be provided to the hydraulic motor chamber so that the driving torque $T_{D}$ overcomes the starting static friction torque $T_{s}$. The right graph of Fig. 2 shows the sinusoidal dither signals with an amplitude $A_{d}$ over the range of the friction torque $T_{f}$ caused by the hydraulic motor. If the sufficient pressure $p_{f}$ corresponding to the starting friction torque $T_{s}$ could not be produced in the motor chambers, the dead zone would appear as follows.

$$
\begin{aligned}
& \left|p_{L}\right| \leq p_{f}: T_{D}=0 \\
& \left|p_{L}\right|>p_{f}: T_{D}=\operatorname{sign}\left(p_{L}\right) D_{m}\left|p_{L^{-}} p_{f}\right|
\end{aligned}
$$

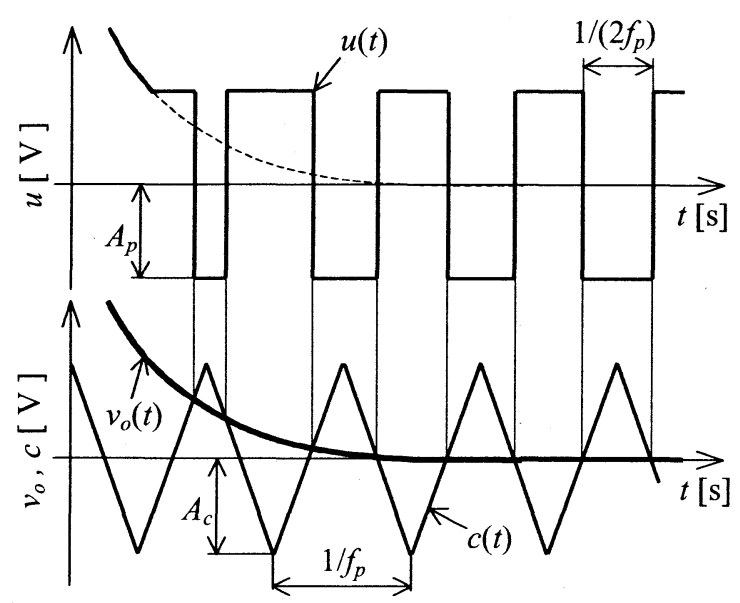

Fig.4 PWM generator
Figure 3 illustrates the block diagram of the system programming. Considering the dead zone and servovalve dynamics, the block diagrams from the servovalve current input to the angular position signal are derived from Eq.(1) as shown in the dotted line. The dynamic parameters and gains in each block are represented by the following equations.

$$
\begin{aligned}
& \tau_{1}=\tau_{2}=C_{v} /\left(K_{p}+1 / R_{m}\right), \quad \tau_{3}=J_{m} / B_{v}, \\
& K_{1}=K_{u} /\left(K_{p}+1 / R_{m}\right), \quad K_{2}=D_{m} /\left(K_{p}+1 / R_{m}\right), \quad K_{3}=1 / B_{v}
\end{aligned}
$$

\subsection{PWM generator}

The use of PWM signal is an effective strategy for removing the non-linearity phenomenon as opposed to the dither method. As shown in Fig.4, translating the input voltage $u$ to a PWM signal with an amplitude $A_{p}$ so as to exceed the friction torque $T_{f}$, the hydraulic motor can be smoothly driven. The PWM signal is generated by comparing the input $v_{o}(t)$ to the triangular carrier signal $c(t)$ with the frequency $f_{p}$. When the input signal $v_{o}$ is larger than the amplitude $A_{c}$ of the carrier wave, the actuating signal $u$ is equivalent to the input signal passing through the PWM generator. The duty ratio of $50 \%$ is adapted when the input signal $v_{o}$ is equal to zero. As shown inside the dashed line in Fig.3, the controller is composed of the Neural Network compensator, reference model, and dither/PWM generator. The original state feedback compensator $\mathbf{F}$ can be replaced with the Neural Network. During the test, it is also possible to arbitrarily select one of the two generators using a switch circuit. The main specification and coefficients on the hydraulic servo system are given in Table 1. 


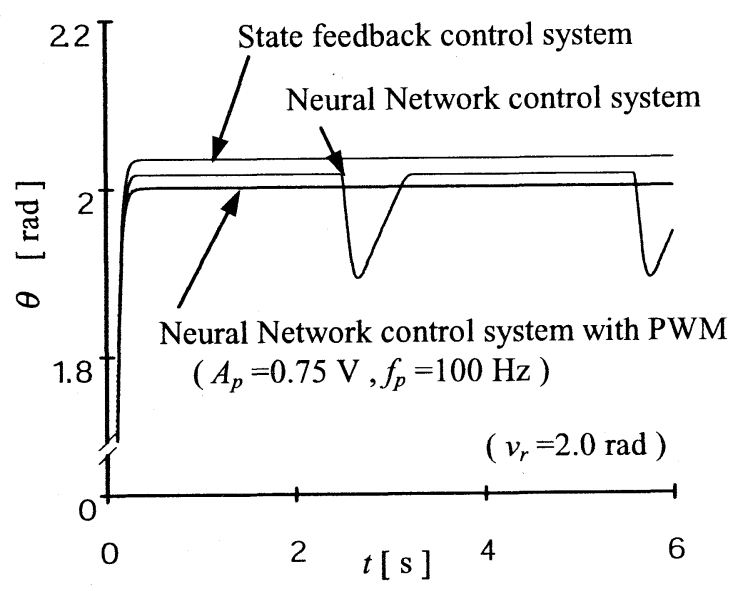

(a) Simulated step response

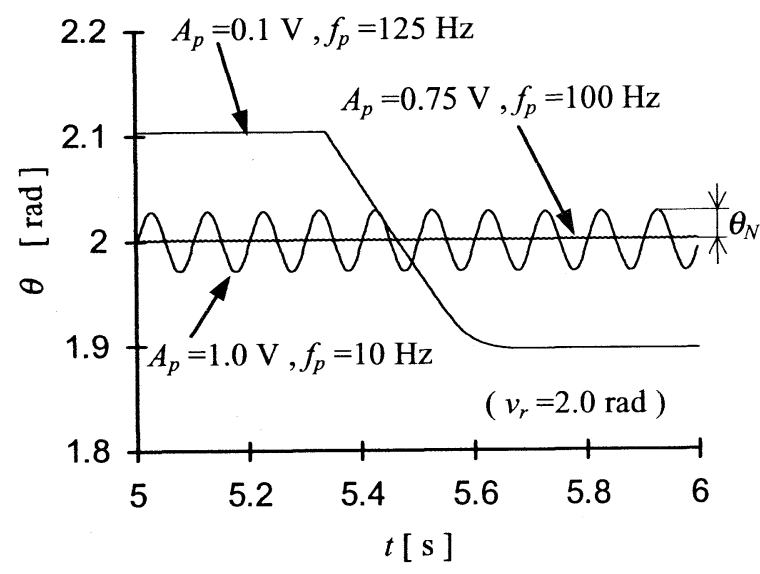

(b) Detail of steady position error (Neural Network control system with PWM)

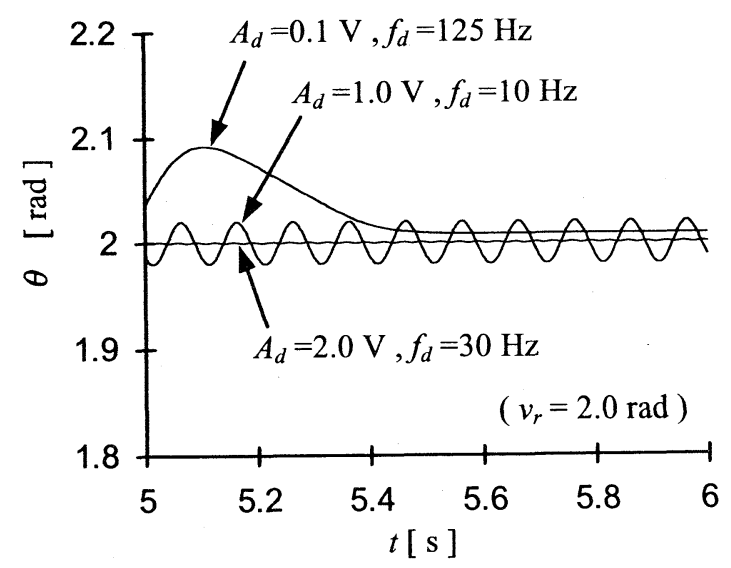

(c) Detail of steady position error (Neural Network control system with dither)

Fig.5 Simulation results of step input
Table 1 Important parameters

\begin{tabular}{|l|l|}
\hline$B_{v}=3.8 \times 10^{-3} \mathrm{Nms} / \mathrm{rad}$ & $K_{p}=9.41 \times 10^{-13} \mathrm{~m}^{5} / \mathrm{Ns}$ \\
\hline$C_{v}=1.24 \times 10^{-14} \mathrm{~m}^{5} / \mathrm{N}$ & $K_{u}=3.24 \times 10^{-5} \mathrm{~m}^{3} / \mathrm{Vs}$ \\
\hline$D_{m}=1.75 \times 10^{-6} \mathrm{~m}^{3} / \mathrm{rad}$ & $T_{s}=2.83 \mathrm{Nm}$ \\
\hline$J_{m}=1.92 \times 10^{-2} \mathrm{kgm}^{2}$ & $T_{k}=0.53 \mathrm{Nm}$ \\
\hline$R_{m}=1.1 \times 10^{11} \mathrm{Ns} / \mathrm{m}^{5}$ & $\tau_{v}=2 \mathrm{~ms}$ \\
\hline
\end{tabular}

\section{SIMULATION RESULTS}

4.1 Steady performance by linear model Typical simulation results are shown in Fig.5. These figures indicate the steady state performance of the step response from 0 to $2 \mathrm{rad}$. Figure 5 (a) shows the differences between the state feedback compensator and Neural Network compensator with and without PWM. It is evident that the application of the PWM signal to the Neural Network control system has an effect on improving the steady error. As detailed in Fig.5 (b) and (c), some steady error and position fluctuation occurs provided that unsuitable amplitudes and frequencies are chosen. On the other hand, setting suitable values, the angular position is well followed with the reference model signal due to the online training of the Neural Network.

These simulation results might be evaluated by two criteria, that is, the amplitude of the noise ripple observed in the steady state position signal, $\theta_{N}$, and the integral of time multiplied by absolute position error, $\Pi$, as shown in the following function.

$$
\Pi=\frac{\int_{t_{1}}^{t_{2}}\left|\theta_{r}(t)-\theta(t)\right| t d t}{\left|v_{r}\right|}
$$

Where, $t_{1}=5 \mathrm{sec}$ and $t_{2}=6 \mathrm{sec}$ are chosen. Figure 6 represents the evaluation chart under the condition of the various frequency $f$ and amplitude $A$. The hatching area

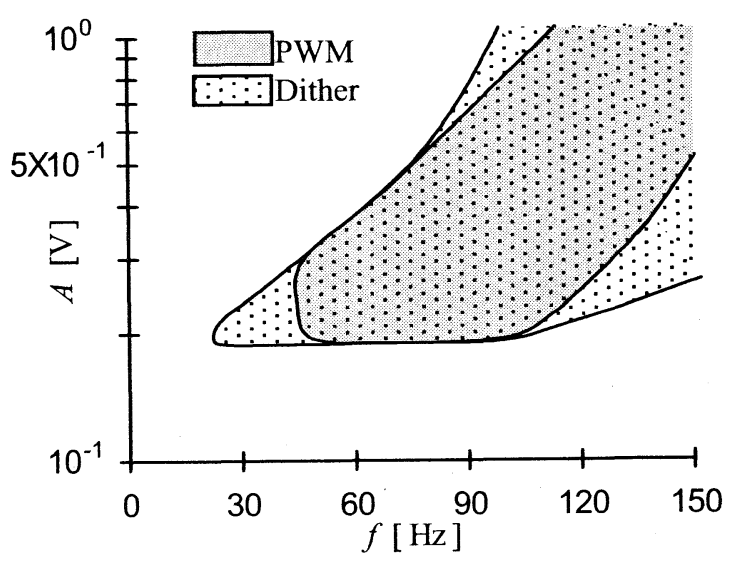

Fig.6. Amplitude and frequency of dither/PWM 
enclosed by the solid curve is determined by $\theta_{N}<0.001$ $\mathrm{rad}$ and $\Pi<0.001$.

It is felt that both areas are similarly distributed in this chart, but the application of the dither signal can be used in a wider frequency and amplitude range. A detailed discussion will be needed for the inside of the border curves in the next step.

\subsection{Static characteristics in non-linear model}

Some simulations are next carried out based on the non-linear mathematical model including the flowpressure characteristics via the valve spool. In order to observe an influence of the dead zone, a series of low frequency sinusoidal waves are given as a reference input. As shown in Fig.7 (a), in the case of the Neural Network control system without dither/PWM technique, the hydraulic motor will not rotate but will keep the static balance where the motor shaft velocity nearly equals zero. It can be seen that adding the dither signal acts as a

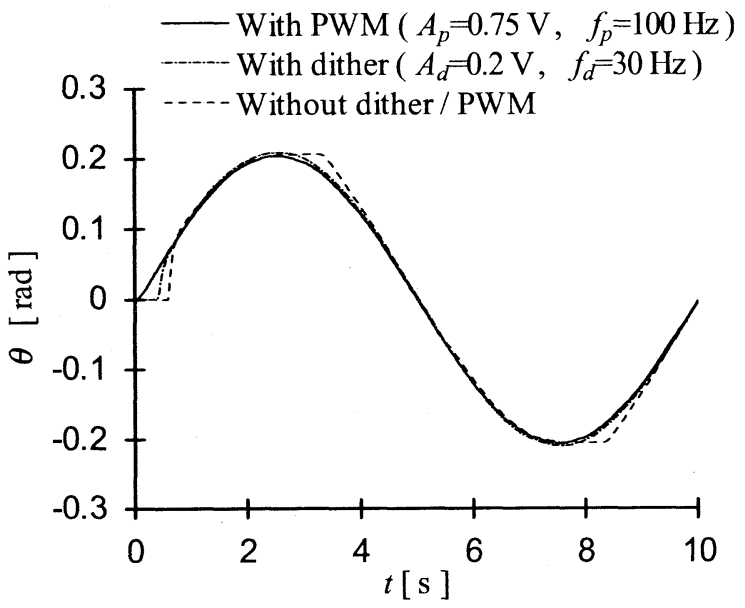

(a) Effect of dither/PWM

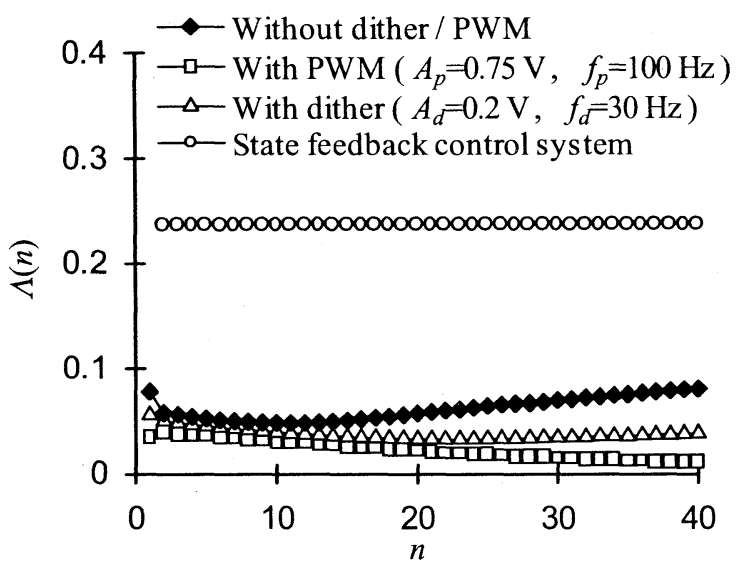

(b) Evaluation values $\Lambda(n)$

Fig.7 Simulation results of sinusoidal input method of effectively supporting the Neural Network online training. On the other hand, as shown in the initial condition $(t=0)$, it is demonstrated that the PWM technique has the excellent advantage of improving the starting characteristics within the dead zone.

Figure 7 (b) represents the learning effect of the Neural Network, while $n=40$ times iteration is performed. The following cost function $\Lambda(n)$ is introduced for evaluating the tracking characteristics.

$$
\Lambda(n)=\frac{\int_{(n-1) / f}^{n / f}\left|\theta_{r}(t)-\theta(t)\right| d t}{\int_{(n-1) / f}^{n / f}\left|\theta_{r}(t)\right| d t}
$$

It is confirmed that the tracking error gradually decreases in the system with the dither/PWM generator, even though it takes a long time. However, there is a tendency that the evaluation value does not level off by only using the Neural Network compensator. Note that the application of the original state feedback compensator could not obtain the appropriate performance compared with the Neural Network control system.

\section{EXPERIMENTS}

\subsection{Test setup}

The experimental apparatus considered in this study is schematically shown in Fig.8. Basically, the test system consists of the hydraulic servo system, digital computer with DSP card and analogue computer. In the PC system, the generation of the pulse signal is restricted to the relatively lower frequency due to the sampling interval 5 msec. Therefore, the wave modulation is made up of the analogue computer with a rapid delay unit; although the pure sinusoidal dither signal $\Delta d$ up to $80 \mathrm{~Hz}$ is simply produced by the digital programming.

The position angle $\theta$, angular velocity $\omega$, and upstream and downstream pressure $p_{1}, p_{2}$ of the motor are detected by sensors. These signals are then sent to the input layer of the Neural Network in the personal computer. The dither or PWM signal is combined with the reference input, and then transmitted to the servo amplifier. Based on the simulation results, the experiments are performed under the condition $f_{d}=30 \mathrm{~Hz}, A_{d}=0.2 \mathrm{~V}$ for the dither

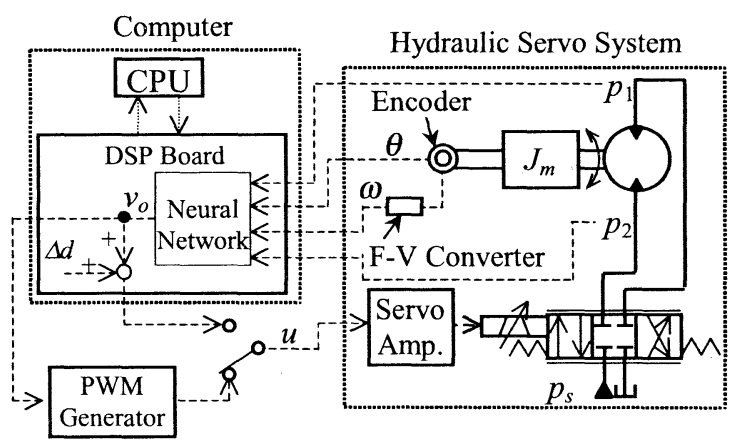

Fig.8 Experimental apparatus 
signal and $f_{p}=100 \mathrm{~Hz}, A_{p}=0.75 \mathrm{~V}$ for the PWM signal. The source pressure $p_{s}$ equals $8 \mathrm{MPa}$, hydraulic fluid with MIL-H-5606C is used and, the oil temperature is kept in 40 degrees Celsius.

\subsection{Experimental results}

Examples of the indicial responses are obtained by applying PWM and dither to the Neural Network control system as shown in Fig.9. It is obviously clear that two techniques are effective for improving the steady error. On the other hand, using only Neural Network compensator, the constant steady errors remain on account of the dead zone. There is a tendency for the PWM method to immediately converge to the reference input compared to the dither signal.

Figure 10 shows the performance index of the steady error which is evaluated by Eq.(5) over the range of the reference input $v_{r}$ from 0.01 to $4 \mathrm{rad}$. Note that only Neural Network control system without dither/PWM is not capable of improving the steady error setting the reference input $v_{r}<=3 \mathrm{rad}$. It can be seen that the evaluation values are almost zero except for the extremely small actuating signal, when the dither/PWM techniques are employed. Especially, using the PWM signal, an excellent performance is completely obtained. It is needless to say that the state feedback compensator without Neural Network has not resulted in good performance of the steady state tracking, even if both countermeasures are taken.

\section{CONCLUSIONS}

This paper suggests a technique that attains precise positioning by using the dither/PWM technique together with the Neural Network compensator in the hydraulic servo motor control system. As a consequence, the simulation and experimental results reveal that the steady errors can be improved by applying the dither/PWM method. It is demonstrated that the PWM technique has slightly better performance in view of the starting torque characteristics. However, it is necessary to assemble more sophisticated circuit components than with the dither. Although the dynamic performance has not been mentioned in this report, it will be presented in the next paper.

\section{REFERENCES}

1. Hibi, A., Torque Performance of Hydraulic Motors at Start and in Low Speed Range, Journal of the JHPS, 7-4, 1976, pp.187-192. (in Japanese)

2. Nakano, K., A Proposal on the Dither, Journal of the Society of Instrument and Control Engineers, 3-4, 1964, pp.262-271. (in Japanese)

3. Ikebe, Y., Sato, S., Inoue, K., Sakamoto, Y, Dither for Improvement of Characteristics of Hydraulic Servomotor in Very Low Speed Region, Transactions of the JHPS, 1973, 4-4, pp.215-223. (in Japanese)

4. Tsai, S. C., Ukrainetz, P. R., Response Characteristics of a Pulse-Width-Modulated Electrohydraulic Servo, Transactions of the ASME, Journal of Basic Engineering, 92, 1970, pp.204-214.

5. Ohuchi, H., Huang, Y., Matsubayashi, T., Fundamental Study of Hysteresis Characteristics of a Direct Drive Electro-Hydraulic Servovalve, Transactions of the JHPS, 31-7, 2000, pp.183-188. (in Japanese)

6. Nishiumi, T., Katoh, H., Konami, S., Application of Neural Network for a Hydraulic Motor/Load System, Transactions of the JSME, 67-659, C, 2001, pp.2212-2218. (in Japanese)

7. Nishiumi, T., Katoh, H., Konami, S., Qausi-Adaptive Position Control of an Electro-Hydraulic Servo Motor System using Neural Network Approach, Proceedings of the 2nd International Workshop on Computer Software Design, Analysis and Control of Fluid Power Systems, 2001, pp.116-123.

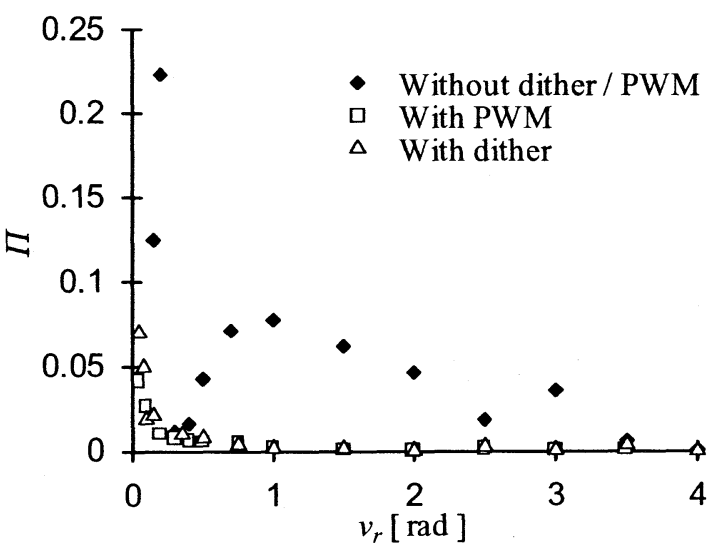

Fig.10 Evaluation of steady error
Fig.9 Experimental results of indicial response

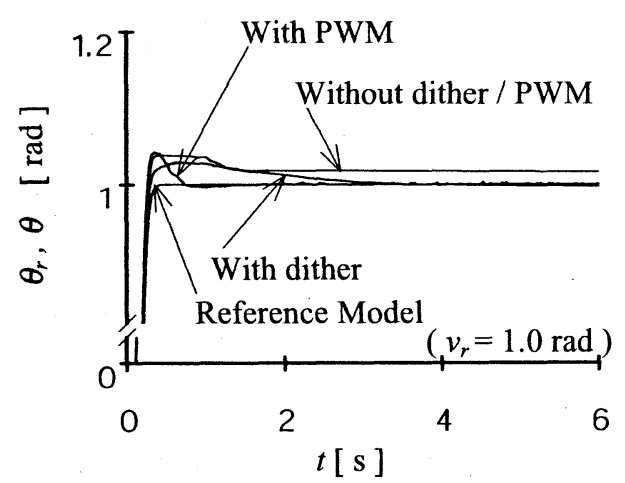

